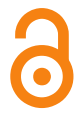

Recibido: 19 septiembre 2019 Revisión: 13 diciembre 2019 Aceptado: 19 junio 2020

\section{Dirección autores:}

Dpto. Didácticas Específicas y Métodos de Investigación y Diagnóstico en Educación. Área Didáctica de la Expresión Musical. Facultad de Ciencias de la Educación. Universidade da Coruña. Campus de Elviña. 15008 A Coruña (España).

E-mail / ORCID

rchao@udc.es

(iD) https://orcid.org/0000-00026584-0049

ruben.vazquez.sanchez@udc.es

https://orcid.org/0000-00020394-8951

\section{a.felpeto@udc.es}

https://orcid.org/0000-00022943-5644
ARTÍCULO / ARTICLE

Audacity como herramienta para la creación de materiales educativos. Una aproximación a través del MOOC «Música para el siglo XXI».

\section{Audacity as a tool for the creation of educational materials. An approach through the MOOC «Music for the 21st century»}

Rocío Chao-Fernández, Rubén Vázquez-Sánchez y Abraham Felpeto-Guerrero

Resumen: En este artículo se analiza la relevancia de Audacity para la creación de materiales educativos, tanto elaborados por docentes, como por estudiantes, ya que posee multiplicidad de funciones y aporta numerosas ventajas, a pesar de su sencillo uso. Para ello, gracias a la creación de un instrumento de análisis de contenido constituido por 8 ítems, se lleva a cabo un estudio de los paisajes sonoros creados por el alumnado del MOOC Música para el Siglo XXI, con el objeto de categorizar los fines educativos de cada uno de ellos y distinguir y caracterizar cada paisaje sonoro según los orígenes de las fuentes sonoras empleadas en función de la clasificación de Schafer (1993). Tras su análisis averiguamos que las aplicaciones didácticas se encuentran dentro de las catalogadas previamente, donde el uso de paisajes sonoros para elaborar tanto recursos musicales propiamente dichos, como relatos y representaciones sonoras a través de construcciones realizadas por el alumnado, ocupan casi el total de las 55 composiciones realizadas en este MOOC. El estudio concluye destacando que el empleo de esta herramienta da soporte a multiplicidad de áreas de conocimiento y sus capacidades como herramienta multitarea ofrece un gran abanico de perspectivas aplicables dentro de los campos de la didáctica específica y la pedagogía.

Palabras clave: Tecnologías de la Información y la Comunicación (TIC), Materiales educativos, Tecnología Educativa, Innovación educativa, Tecnología de código abierto.

Abstract: This article analyses the relevance of Audacity as a creation tool of learning materials for both teachers and students, since it has multiple functions and provides numerous advantages, despite its simple use. To that purpose, thanks to the creation of an specific content analysis tool based in 8 items, an study of soundscapes created by the students of the MOOC Música para el Siglo XXI is carried out with the purpose of categorize their educative aims as well as distinguish and characterize each soundscape according with the origin of their sound source in relation with the classification proposed from Schafer (1993). Thanks to the latter analysis we know that the didactic applications are within those previously catalogued, where the use of soundscapes both to elaborate musical resources as well as to create sound representations through constructions carried out by the students make a clean sweep of almost all of the 55 analyzed compositions. The study concludes with the idea that this tool gives support to multiple areas of knowledge and that its capabilities as a multitasking tool provide a wide range of perspectives applicable within areas such as pedagogy or specific didactic fields.

Keywords: Information and Communication Technologies (ICT), Educational Resources, Educational Technology, Educational innovation, Open Source Technology. 


\section{Introducción}

El empleo de herramientas TICs para la creación o la actualización de nuevos materiales educativos son un continuo desde la entrada de internet en el aula. Las tendencias y el uso de las aplicaciones van variando con el tiempo y, en función de su popularidad o sus propuestas formativas o educativas, se van implementando poco a poco en las clases. Sin embargo, algunas se han mantenido estables en el tiempo, como Audacity. Aunque esta aplicación multiplataforma de software libre, cuya función principal es la creación y edición de audio, tiene su origen en 1999, es desde el año 2004 cuando empiezan a aparecer artículos en revistas de divulgación en los que se realizan tutoriales y explicaciones sobre su uso -véase Saulo Bolaños (2004)- pasando a centrarse poco a poco las publicaciones del ámbito investigador de los años posteriores en un empleo más extenso de la aplicación desde una perspectiva de uso aplicada al aula, como podemos observar en publicaciones como las de Smith (2011), Ponce y Lago (2017) o Sales, Roig y Marín (2019), entre otras. Ello radica en que todas las funcionalidades que posee la aplicación facilitan su empleo por parte de los docentes para incorporar y elaborar materiales didácticos $y$, al tiempo, resulta excelente para que el alumnado comience a explorar por sí mismo las oportunidades que ofrece, siendo aplicables en el aula de educación musical o en cualquier otra área.

\subsection{Audacity como herramienta para la creación de material educativo}

A pesar de ser una aplicación orientada hacia la edición de sonido, ofrece posibilidades muy amplias, encontrando investigaciones en las que se emplea desde muy diversos enfoques, muchos no ligados al ámbito de la educación musical, sino abordados desde una perspectiva interdisciplinar, formando en ocasiones parte del tema central de la investigación, utilizándose otras veces como herramienta en el proceso investigativo. Por ello, enumeraremos y clasificaremos algunos fines para los que se ha empleado la aplicación y citaremos algunas investigaciones recientes para ver el proceso de implementación de ésta dentro de la actividad realizada.

\section{Usos genéricos}

Dentro de los usos genéricos más comunes se encuentra la edición de sonido propiamente dicha; tal y como se aprecia en los numerosos manuales y tutoriales existentes en repositorios educativos, como INTEF -intef.es- o los repositorios institucionales de muchas de las consejerías de educación de España. Todos ellos se centran en el proceso de instalación de la aplicación, así como en la explicación de sus diferentes opciones desde el punto de vista de un trabajo puramente mecánico de edición, conversión o grabación de audio, sin proponer un uso concreto del software, más que guardar el resultado de la edición/grabación.

\section{Diseño de paisajes sonoros en educación musical}

Para comprender qué uso puede tener Audacity en el diseño de paisajes sonoros, es importante comenzar contextualizando este término, propuesto por Murray Schafer en 1969 como derivación del vocablo inglés "soundscape"; diferenciándose del mismo en que no tiene por qué estar limitado únicamente a lugares exteriores. Así, paisaje sonoro es todo el entorno acústico que nos rodea (Ferretti, 2006). 
La relación entre el paisaje sonoro y Audacity es realmente estrecha. Se puede abordar el trabajo del paisajismo sonoro fundamentalmente desde dos ejes; formando, además, parte inseparable del currículum de educación musical dentro de la etapa de educación primaria resultando en percepción y expresión (Palmese, Carles y Alcázar, 2010). Respecto a la percepción, Audacity permite escuchar y aislar fragmentos de audio grabados por nuestro alumnado durante una salida de campo o paseo sonoro, de forma que posteriormente sea posible analizar, enumerar, comentar, describir, etc. los sonidos que albergan las grabaciones para crear diarios de campo, guías y mapas sonoros, seleccionar fragmentos para realizar futuras composiciones, establecer similitudes y diferencias entre distintos ambientes sonoros, entre otros. Respecto a la expresión, existe igualmente un abanico muy amplio de posibilidades tales como componer un paisaje sonoro imaginario con fragmentos seleccionados anteriormente, musicar historias o cuentos, descontextualizar paisajes sonoros introduciendo elementos ajenos a ellos, o distorsionando los existentes y un largo etcétera. Se nos ocurre igualmente que desde el punto de vista de la expresión musical y corporal podemos introducir la interpretación de fragmentos musicales cantados (Sichivitsa, 2007) como paisajes sonoros improvisados, de forma que su uso se centre en emplear esta herramienta para eliminar aquellas variables que pudiesen minimizar el rendimiento en el aula, como la vergüenza que puede causar al alumnado cantar un fragmento musical delante de sus compañeros $y$, al tiempo, promover nuevas áreas como la experimentación y descontextualización de sonidos a través de la grabación de la propia voz aplicando diferentes efectos, o potenciar la improvisación vocal jugando con el método grabación-audición para analizar los fallos cometidos. Como se puede observar, el uso del paisaje sonoro como material educativo abre un rico abanico de posibilidades desde multiplicidad de perspectivas.

Como ejemplo de ello, podemos hallar numerosas investigaciones o proyectos en los que se utiliza Audacity para crear paisajes sonoros con diferentes finalidades. Desde el punto de vista de la reminiscencia Lee et al. (2015) proponen la creación de paisajes sonoros como forma de comunicar las conclusiones de una investigación de corte histórico sobre una localidad, donde los sonidos actúan como una interpretación acústica de las evidencias históricas encontradas.

En la misma línea encontramos investigaciones como las de Sikora, Grčić y Russo (2016), donde se utiliza Audacity -junto a otras aplicaciones- para crear paisajes sonoros que serán utilizados por turistas a modo de realidad de audio aumentada por medio de dispositivos móviles y geolocalización, para que puedan comprender mejor la historia del lugar a lo largo del tiempo. Asimismo, Ariza-Pomareta (2015) realiza una propuesta de paisaje sonoro como cápsula del tiempo, a partir de la realización y grabación de paseos sonoros y salidas de campo.

Desde el punto de vista del análisis, ejemplos como el expuesto por Ibáñez Barrachina y De Vicente-Yagüe Jara (2014), que usan la aplicación como herramienta de análisis y de registro, con el fin de conocer las frecuencias de las notas para posteriormente comprobar las desviaciones existentes en las interpretaciones de los instrumentos con el fin de enseñar al alumnado -o realizar una práctica- sobre precisión en la afinación de un instrumento o de la propia voz, pueden extrapolarse al paisajismo sonoro para comprobar la forma en la que va cambiando un determinado lugar a lo largo del tiempo, pudiendo así el alumnado establecer diferencias y similitudes de un medio ambiente sonoro por ejemplo a lo largo de las diferentes estaciones del año. 
Ya desde una perspectiva más lúdica, podemos descubrir experiencias como la composición de paisajes sonoros de terror para videojuegos o películas con la intención crear patrones sonoros emocionales que creen una percepción de inmersión dentro de una atmósfera tensa (Lopes, Liapis y Yannakis, 2017).

\section{Herramienta pedagógica o didáctica}

En este estudio no solo se ha puesto el foco en el propio software per se, sino en las experiencias que surgen a través de este, para la implantación de acciones formativas y pedagógicas que sirven para ejemplificar, mostrar o demostrar eventos desde una perspectiva didáctica y desde multiplicidad de áreas. En ellas, Audacity se explota, entre otros usos, como analizador de espectros; lo cual servirá para, a través de ejemplos, desarrollar la comprensión física de un fenómeno sonoro en alumnado universitario (Gailey, 2015) o de efectos sonoros concretos, como el efecto Doppler (Dias, Carvalho y Rodrigues, 2016), en áreas como la física.

En educación física se utiliza Audacity para preparar las secuencias musicales que acompañan los diferentes tipos de actividad, mezclando así en una misma línea temporal de audio diversos fragmentos con diferencias significativas de tempo entre ellos, permitiendo así hacer cambios de intensidad en la práctica deportiva (Karageorghis, 2017).

En la enseñanza de lenguas Audacity es muy utilizado por el profesorado para crear karaokes, de forma que puedan eliminar de un fragmento musical las frecuencias con voz, para después poder reproducir el fragmento y que el alumnado practique la dicción y gane confianza en la pronunciación a través del canto (Sánchez-Satoca, 2012) o también creando entrevistas a personas que hablen una determinada lengua con la finalidad de poder reproducirlas a posteriori para practicar la audición y pronunciación (Marson y Santos, 2010).

También es posible utilizar Audacity en la enseñanza de las matemáticas haciendo zoom sobre una pista de audio hasta llegar a ver sus ondas. Así, el alumnado de bachillerato o universidad podrá comprobar que la forma que estas ondas tienen no es sino una representación de las funciones que han estudiado en clase (Godofredo, León y Liern, 2010) o estudiar fenómenos físicos relacionados con el sonido (Beltrán y Rodríguez, 2017).

Igualmente, y de forma interdisciplinar, puede plantearse el uso de Audacity para la creación de Podcasts educativos donde el producto obtenido es una grabación con contenidos elaborados por profesores, haciendo que el tiempo y el espacio sean flexibles en la enseñanza a través de la inserción de diversas opciones de recursos didácticos digitales y que estimulan el proceso de enseñanza para potenciar el aprendizaje en los estudiantes. Ejemplos de esto se muestran en las investigaciones de Bairros-Soares, Vielmo-Miranda y Barin-Smaniotto (2018) o Amundsen y Belmonte (2018), donde exponen los beneficios de ofrecer un contenido complementario, motivador y que potencia el aprendizaje o las de Bell y Buri (2017) o Galán Camacho (2018) donde explican cómo emplear Audacity para la creación de un podcast.

También puede utilizarse a partir de una creación del alumnado, generándolo en base a experiencias previas como consumidores y productores y aplicando los conocimientos y competencias que han adquirido en la escuela, resultando así un producto propio, en un formato diferenciado (Forbes y Khoo, 2015). 


\section{Creación de narraciones, historias animadas o de realidad aumentada}

Aunque hasta ahora se había atendido a experiencias narrativas solamente auditivas los podcasts-, también se elaboran contenidos audiovisuales de mayor tamaño que los anteriores, que sirven como ejemplo para el profesorado y para el alumnado. Wakefield y France (2015) emplean las historias digitales para la creación de entornos audiovisuales donde el alumnado utiliza este programa para la elaboración de contenidos sirviendo a la vez para evaluarlos, al tiempo que conforman sus actividades de campo. De esta forma, los diversos contenidos son integrados a través de una misma herramienta, al menos en el área sonora.

Existen otras experiencias de creación de materiales didácticos de última generación, como la Realidad Virtual o Realidad Aumentada, en las que además de la elaboración de los contenidos puramente visuales, se suelen añadir una o varias capas de sonido para dotar de mayor verosimilitud al escenario que se está componiendo en el caso de la Realidad Virtual- o para añadir información significativa al entorno u objetos que se están aumentando (Nielsen, Brandt y Swensen, 2018). Experiencias e investigaciones piloto de diseño de este tipo de materiales didácticos por profesorado y alumnado los encontramos en proyectos de diferentes ámbitos, como la enseñanza de ciencias en el proyecto ArSci (Lund, Brandt y Swensen, 2018) o la didáctica del paisaje en el proyecto L'Hort (Hurtado y Botella, 2017).

\section{Método}

Habiendo planteado un marco para el contexto de esta investigación, los objetivos que nos planteamos son categorizar los fines educativos de cada uno de los paisajes sonoros y distinguir y caracterizar cada paisaje sonoro según los orígenes de las fuentes empleadas en función de la clasificación de Schafer (1993). Para alcanzar dichos objetivos, se expondrá la caracterización de la muestra, los ítems utilizados en el instrumento de análisis creado y los análisis pertinentes.

\subsection{Muestra}

Como muestra para esta investigación se emplean las creaciones sonoras compuestas por parte de alumnado del MOOC Música para el siglo XXI en forma de paisajes sonoros, dentro del marco de la actividad de evaluación final por pares, paisajes que deberían satisfacer al menos las siguientes premisas: contener varias secuencias sonoras diferentes -pudiendo sonar o no simultáneamente-; emplear, al menos, un efecto proporcionado por el software y por último poseer una duración próxima a un minuto.

Dicho MOOC, se concibe desde el contexto de la tecnología como generadora de material didáctico de calidad adaptado a las necesidades del alumnado (Cepeda, Gallardo y Rodríguez, 2017), más que como herramienta propiamente dicha y desde un escenario de falta de conocimiento por parte del profesorado de las posibilidades que aportan las TIC a la educación musical (Chao-Fernández, Felpeto-Guerrero y VázquezSánchez, 2018). Estructurado en cuatro módulos independientes, el primero aborda una aproximación al concepto de competencia mediática y a las aportaciones del software libre a la educación musical. En los tres restantes se presentan tres aplicaciones software básicas en la formación musical: LenMus, MuseScore y Audacity. 
A mayores, se incluye un quinto módulo a modo de actividad final de la que se seleccionarían los paisajes sonoros.

Esta selección resulta de interés ya que durante el MOOC se forma al alumnado en el uso de software pedagógico-musical y, al elaborar dicha actividad final se estarían integrando en ella los conocimientos -tanto musicales como de uso del softwareadquiridos durante el MOOC así como sus conocimientos previos, obteniendo una muestra válida de 55 paisajes sonoros.

\subsection{Instrumentos}

Inicialmente, la información dada por el alumnado participante se obtiene de la ficha que han de aportar junto al paisaje sonoro, donde deben exponer su título, descripción, clips de sonido empleados, efectos y bancos de sonidos.

De cara al análisis, se creó un instrumento propio basado en las técnicas de análisis de contenido (Krippendorff, 1997) -sirviéndonos del desarrollado por Moscoso, Peck y Eldridge (2018)-, abierto a más variables. Constituido por ocho ítems de respuesta abierta, unos de carácter cuantitativo -número de sonidos, número de efectos empleados y la duración en segundos- y otros cualitativo: descripción de los sonidos utilizados, tipo de efecto/s empleado/s, banco/s de sonidos de los que los han extraído, tipo de fuente sonora o de comunidad en la que se emplean y palabras clave-; que servirán para establecer diferentes niveles de relación entre ítems.

\subsection{Procedimiento}

Para comenzar, se descargaron todos los archivos obtenidos a partir de la actividad realizada -junio y julio de 2018-, cribados según las premisas anteriormente descritas. Se analizaron todos los paisajes sonoros resultantes teniendo en cuenta la ficha descriptiva que cada alumno/a anexó al archivo de audio de su paisaje sonoro compuesto, siendo organizados según la clasificación del sonido de Schafer (1993), modificada para alcanzar los objetivos de esta investigación.

Una vez volcados los datos, se realiza un análisis cuantitativo -tablas de frecuencia- y establecen relaciones entre los datos establecidos anteriormente-a través de los resultados de las tablas de frecuencia y la inferencia de estos datos con los extraídos a través del análisis de contenido-. Para ello, se emplea el software de análisis estadístico SPSS, versión 23.

\section{Resultados}

\subsection{Descripción general del total de la muestra}

Se realiza un análisis descriptivo del total de la muestra (55 paisajes sonoros), en los que se hayan desde 3 sonidos por cada paisaje -dos casos- hasta los 16 -dos casos-, siendo 5 el número de sonidos que define la moda de este análisis -21 casos-.

El número de efectos empleados por cada paisaje sonoro varía de entre el uso de un solo efecto -cinco casos- hasta los nueve -un caso-, siendo lo más habitual el uso de cuatro en cada una de las obras -14 casos-. Si bien se han analizado en función de su cantidad, también se ha realizado un análisis por categorías, donde encontramos 
28 tipos de efectos diferentes. El más empleado es Desvanecer, seguido por Aparecer, Panoramización, Reverb, Amplificar, Eco y cambio de velocidad. Para conocer su distribución en porcentajes, puede ver el gráfico 1.

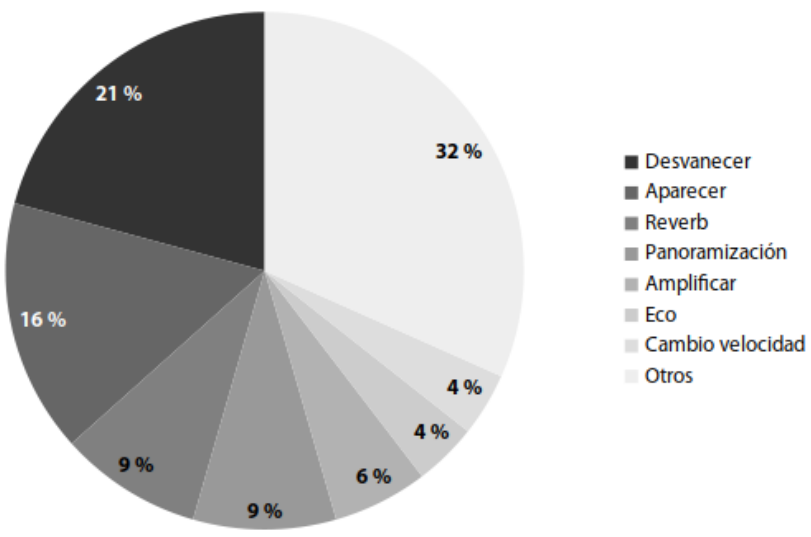

Gráfico 1. Frecuencia de uso en porcentaje de los efectos empleados en los paisajes sonoros. Fuente: Elaboración propia

Con el origen de los bancos de sonidos empleados para la creación de los paisajes sonoros, destacan dos, el banco del INTEF -https://intef.es/recursoseducativos/banco-de-imagenes-y-sonidos/- y el de la web Freesound.org. Con un menor porcentaje de aparición están el BBC Sound Effects -bbcsfx.acropolis.org.uk-, RecursosTIC -recursostic.educacion.es- y las webs Sshhtt -sshhtt.com- y YouTube youtube.com-. Por debajo de estos, el uso de otros bancos aparece en una cuantía menor al 4\%. Por último, catorce de los participantes importan sonidos de bancos propios o autoelaborados (Gráfico 2).

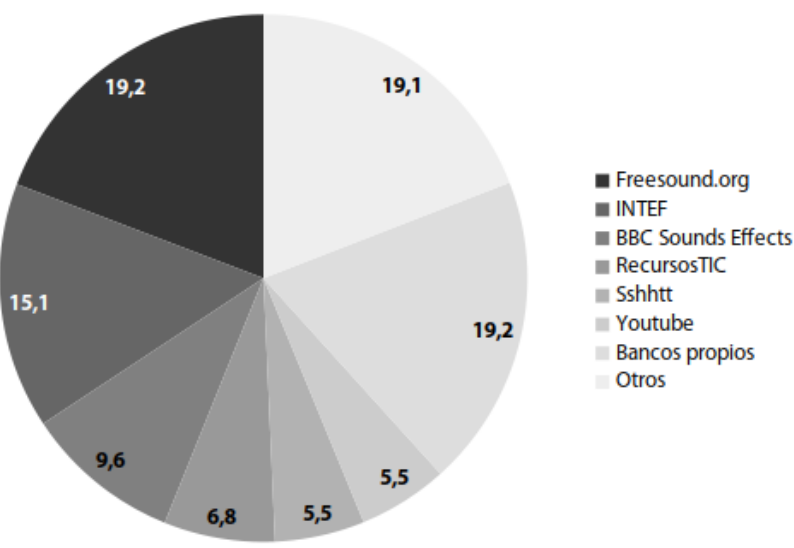

Gráfico 2. Frecuencia de uso en porcentajes de los efectos disponibles en Audacity. Fuente: Elaboración propia

En cuanto a la duración de los paisajes sonoros, destaca su mayor heterogeneidad en comparación con los ítems analizados anteriormente, encontrando duraciones de entre 34 y 126 segundos, siendo 60 la duración más comúnmente empleada. 


\subsection{Análisis y categorización según el fin para el que se crea cada paisaje sonoro}

Una vez señaladas las características generales del total de las creaciones analizadas, procedemos al análisis cuantitativo de la muestra. Para ello, se presentará una clasificación de los diferentes paisajes sonoros en función de su fin, divididos tras el establecimiento de palabras clave como descriptoras y los diferentes fragmentos sonoros obtenidos. Tras el análisis y la creación de categorías, se establecen los siguientes fines y sus parámetros:

- Composición musical: Todas aquellas grabaciones que poseen, tanto cuantitativa como cualitativamente, una mayoría de sonidos o fragmentos musicales.

- Representación de un paisaje sonoro real: Aquellas grabaciones que tratan de recrear una toma en un ambiente real y que no poseen intencionalidad alguna de recrear acciones o secuencias de forma ordenada y con un fin argumentativo.

- Relatos sonoros: Al contrario que los anteriores, los autores tratan de establecer una secuencia argumental a través de sonidos que poseen inicio, nudo y desenlace.

- Recreación de un fragmento sonoro de realidad aumentada: Grabación en la que el autor ejemplifica, a través del sonido, todas aquellas acciones que va realizando con las posibilidades que le ofrece la aplicación.

Si bien la clasificación establecida para estas cuatro categorías es clara, es importante tener en cuenta que la representación de un paisaje sonoro real estaría más próxima al concepto de paisaje sonoro de Schafer (Ferretti, 2006) o a ámbitos como el de la biología o la ecología. La categorización de relatos sonoros estaría más próxima al de las narraciones sonoras, donde tratan de realizar una producción sonora socioambiental para la construcción de una audición inteligente, aplicada a la explicación de sucesos o acontecimientos reales, similares a la experiencia realizada por Silva (2011). En la tabla 1 se aprecian los resultados.

Tabla 1. Cuantificación en función de los fines para el que es creada el paisaje sonoro.

\begin{tabular}{lccc}
\hline & Frecuencia & Porcentaje & $\begin{array}{c}\text { Porcentaje } \\
\text { acumulado }\end{array}$ \\
\hline Composición musical & 7 & 12,7 & 12,7 \\
Composición musical con sonidos naturales & 1 & 1,8 & 14,5 \\
Realidad aumentada & 1 & 1,8 & 16,4 \\
Relato sonoro & 25 & 45,5 & 61,8 \\
Representación sonora & 21 & 38,2 & 100,0 \\
& & 100,0 & \\
\hline
\end{tabular}

Fuente: Elaboración propia.

\subsection{Caracterización de los paisajes sonoros en función del fin para el que se crea}

A continuación, comenzaremos con la distribución de cada fuente sonora -entendida como cada uno de los sonidos individuales presentes en cada creación- organizadas 
en función de cada uno de los fines para los que ha sido construido cada paisaje sonoro, y que constituyen un valioso material educativo. Se partirá de la explicación de las creaciones categorizadas como composiciones musicales.

\section{Composiciones musicales}

Aquí agrupamos los paisajes sonoros categorizados como composiciones musicales propiamente dichas, así como aquellos paisajes sonoros categorizados como una composición musical que intercala sonidos naturales. Dentro de estos ocho paisajes sonoros predomina la aparición de "grabaciones de música" -sonidos clasificados así según el instrumento de medida empleado- apareciendo éstas entre 1 y 8 ocasiones dentro de cada uno de los paisajes, además de incluir también "sonidos de instrumentos" -en este caso, musicales- presentes en este caso en 3 paisajes sonoros entre 1 y 4 sonidos por paisaje sonoro-, aunque también encontramos 3 paisajes sonoros en los que aparecen sonidos del bosque o naturaleza y sonidos marinos, de pájaros, de insectos, voces humanas incomprensibles, sonidos de ceremonias y otros entretenimientos (una sola muestra sobre el total contabilizado). Sin embargo, todos aquellos sonidos que no son categorizados como sonidos de música ya compuesta o instrumentos musicales, son arrojados de dos creaciones sonoras: la primera es la que recrea un ambiente natural de fondo y la otra es una composición artística musical que mezcla música y otros sonidos humanizados.

\section{Realidad aumentada}

El único paisaje sonoro categorizado como realidad aumentada es el que emplea 4 pistas de audio donde el autor explica qué hará con su voz, qué efecto aplicará y el resultado de este tras haberlo dicho, con una música de fondo que se mantiene invariable durante toda la grabación.

\section{Relatos sonoros}

En tercer lugar, tenemos los relatos sonoros. Lo componen un total de 25 paisajes sonoros en los que los autores recrean una narración a través del sonido. En este apartado existe una mayor variabilidad en cuanto a las categorías establecidas por Schafer (1993) y recogidas aquí.

Para empezar, comenzamos con la categoría sonidos naturales. Se emplean, principalmente, sonidos relacionados con de agua y truenos (5 casos) y de mamíferos salvajes -4 casos-. En los sonidos generados por personas, donde los sonidos del cuerpo -como son los pasos de una persona o latidos del corazón, con 21 apariciones en los 25 relatos sonoros; las voces humanas, 10 casos y los gritos y lloros, en 5 ocasiones- son los que ofrecen una incidencia mucho mayor que en el resto de las categorías. En la categoría sonidos y sociedad, destacan de sobremanera los sonidos relacionados con las profesiones, la vida diaria o los negocios, con 12 apariciones dentro de los 25 paisajes contabilizados; seguidamente tenemos 9 apariciones de sonidos relacionados con medios urbanos o de una ciudad y 8 apariciones categorizadas como música incidental de fondo, para crear una atmósfera para la acción. Relegados al cuarto puesto se sitúan las 4 apariciones de animales domésticos y los sonidos relacionados con festividades o ceremonias.

En la categoría de sonidos mecánicos, localizamos 14 apariciones de sonidos de herramientas o utensilios mecánicos (tabla 2), destacando 9 casos de sonidos de 
bisagras de puertas, compartiendo 5 casos los sonidos de motores de combustión interna y los vehículos de transporte -automóviles y motocicletas-. En la penúltima categoría, la de sonidos como indicadores, las campanas y los sonidos de sistemas de alerta o alarmas, copan las dos primeras posiciones, con 5 casos, sucediéndole los sonidos de teléfonos, con 4 casos. Finalmente, en la última categoría -Otros-, encontramos 2 sonidos fuertes, cuyo origen no es facilitado por el alumnado y no es posible situarlo en otra categoría.

\section{Representaciones sonoras}

Por último, se describirá la categoría de representación de un paisaje sonoro real en función de los sonidos empleados para su construcción. En el apartado de sonidos naturales destaca que, a excepción de los sonidos de reptiles y fuego, se obtiene que existe al menos algún caso en cada uno de ellos. Destacan los sonidos del agua y de pájaros -ambos con 11 casos-; seguidos por los sonidos de mamíferos salvajes -5 casos- y los sonidos de viento y aire -4 casos-. En la categoría de sonidos humanos, aparecen, principalmente, sonidos del cuerpo -9 casos - y voces humanas -5 casos de voces comprensibles y 2 de voces incomprensibles-. Aquellos que son categorizados como sonido y sociedad, predominan los sonidos de paisajes marinos -6 casos- y de animales domésticos -5 casos-; siguiéndoles los sonidos de entretenimientos diferentes a las redes sociales y los paisajes domésticos, sonidos de profesiones, vida diaria o de negocios -3 casos-. En la categoría de sonidos mecánicos, destacan los sonidos de máquinas de transporte -4 ocasiones-, seguidas de sonidos de trenes -2 ocasiones- y los de herramientas o utensilios mecánicos y, por último, los sonidos de aviones o avionetas, armas y de motores de combustión interna -con una sola aparición en cada caso-. En los sonidos como indicadores, en primer lugar, se sitúan los sonidos de alarmas y alertas -con 3 casos-, seguidos de campanas e instrumentos -en este caso, musicales, con 2 casos en ambas subcategorías- y de alertas de medios de comunicación -un solo caso-. No hay ningún sonido que no pudiese ser clasificado.

\subsection{Agrupación de cada fuente sonora en función de la clasificación de Schafer}

Si realizamos un análisis en función del total de sonidos empleados, encontramos que los más empleados en los paisajes sonoros son sonidos del agua -16 paisajes contienen este tipo de sonidos-, de pájaros -15 paisajes- y de mamíferos -9 paisajesdentro de la categoría de sonidos naturales. Dentro de los sonidos humanos, si bien destacan los sonidos del cuerpo -apareciendo en 30 paisajes-, también hay un número importante de voces humanas -19 en total, siendo 14 de voces humanas comprensibles y 5 de voces humanas incomprensibles-y de lloros/gritos -6 paisajes-.

En la categoría de sonidos y sociedad, destaca el empleo de la música, en un total de 18 paisajes, y de paisajes urbanos como cantidad más destacable (10 paisajes los emplean), así como de animales domésticos, que aparecen en 9 paisajes sonoros. Como sonidos mecánicos, hallamos que los participantes prefieren los de herramientas o utensilios mecánicos y de medios de transporte y son los que copan esta categoría en 16 y 9 ocasiones, respectivamente-. Dentro de sonidos como indicadores, el uso de alarmas o sistemas de alerta, junto con campanas y gongs e instrumentos sonoros son los sonidos más destacables en esta categoría, presentándolos en 8, 7 y 6 paisajes, respectivamente. Por último, en la categoría establecida por Schafer (1993) como Otros, hallamos la concurrencia de dos sonidos fuertes cuyos autores no explican su origen y no son clasificables dentro de ninguna otra categoría (Tabla 2). 
Tabla 2. Tabla de frecuencias basada en la clasificación de Schafer (1993) que muestra tanto la categoría asignada como el tipo de fin del paisaje sonoro.

\begin{tabular}{|c|c|c|c|c|c|c|c|}
\hline & & $\begin{array}{l}\text { Comp. } \\
\text { musical }\end{array}$ & $\begin{array}{l}\text { Comp. } \\
\text { musical/ } \\
\text { sonidos } \\
\text { naturales }\end{array}$ & $\begin{array}{c}\text { Realidad } \\
\text { aumentada }\end{array}$ & $\begin{array}{l}\text { Relato } \\
\text { sonoro }\end{array}$ & $\begin{array}{l}\text { Represen- } \\
\text { tación } \\
\text { sonora }\end{array}$ & $\begin{array}{c}\text { Total } \\
\text { según } \\
\text { el tipo } \\
\text { de } \\
\text { fuente } \\
\text { sonora }\end{array}$ \\
\hline \multirow{14}{*}{$\begin{array}{l}\text { Sonidos } \\
\text { naturales }\end{array}$} & Agua & & & & 5 & 11 & 16 \\
\hline & Aire & & & & 1 & 4 & 5 \\
\hline & $\begin{array}{l}\text { Bosque, selva, } \\
\text { naturaleza }\end{array}$ & 1 & & & 3 & 3 & 7 \\
\hline & Fuego, Ilamas & & & & & & \\
\hline & Pájaros, aves & 1 & 1 & & 2 & 11 & 15 \\
\hline & Felinos salvajes & & & & & 1 & 1 \\
\hline & Insectos & & 1 & & & 2 & 3 \\
\hline & Mamíferos & & & & 4 & 5 & 9 \\
\hline & Reptiles & & & & & & \\
\hline & Animales (otros) & & & & 1 & 1 & 2 \\
\hline & $\begin{array}{l}\text { Estaciones, } \\
\text { noche-día }\end{array}$ & & & & 1 & 2 & 3 \\
\hline & Hojarasca o árboles & & & & 1 & 1 & 2 \\
\hline & Truenos, tormenta & & & & 5 & 1 & 6 \\
\hline & Total & 2 & 2 & 0 & 23 & 42 & 69 \\
\hline \multirow{5}{*}{$\begin{array}{l}\text { Sonidos } \\
\text { humanos }\end{array}$} & $\begin{array}{l}\text { Voces humanas } \\
\text { comprensibles }\end{array}$ & & & 1 & 8 & 5 & 14 \\
\hline & $\begin{array}{l}\text { Voces humanas } \\
\text { incomprensibles }\end{array}$ & 1 & & & 2 & 2 & 5 \\
\hline & Gritos, lloros & & & & 5 & 1 & 6 \\
\hline & Sonidos del cuerpo & & & & 21 & 9 & 30 \\
\hline & Total & 1 & 0 & 1 & 36 & 17 & 55 \\
\hline \multirow{13}{*}{$\begin{array}{l}\text { Sonidos y } \\
\text { sociedad }\end{array}$} & Paisaje rural & & & & 1 & 1 & 2 \\
\hline & Paisaje de villa & & & & & & \\
\hline & Paisaje urbano & & & & 9 & 1 & 10 \\
\hline & Paisaje marino & & 1 & & 1 & 6 & 8 \\
\hline & Paisaje doméstico & & & & 1 & 2 & 3 \\
\hline & $\begin{array}{l}\text { Sonido de profesio- } \\
\text { nes, negocios y vida } \\
\text { diaria }\end{array}$ & & & & 12 & 3 & 15 \\
\hline & Animales domésticos & & & & 4 & 5 & 9 \\
\hline & $\begin{array}{l}\text { Televisión, radio o } \\
\text { películas }\end{array}$ & & & & 2 & & 2 \\
\hline & $\begin{array}{l}\text { Sonidos de redes } \\
\text { sociales }\end{array}$ & & & & & & \\
\hline & $\begin{array}{l}\text { Sonidos de otros } \\
\text { entretenimientos }\end{array}$ & 1 & & & 3 & 3 & 7 \\
\hline & Música & 6 & 1 & 1 & 8 & 2 & 18 \\
\hline & $\begin{array}{l}\text { Ceremonias o } \\
\text { celebraciones }\end{array}$ & 1 & & & 4 & 1 & 6 \\
\hline & Total & 8 & 2 & 1 & 45 & 24 & 80 \\
\hline
\end{tabular}

Fuente: Elaboración propia. 
Tabla 2. (Cont.) Tabla de frecuencias basada en la clasificación de Schafer (1993) que muestra tanto la categoría asignada como el tipo de fin del paisaje sonoro.

\begin{tabular}{|c|c|c|c|c|c|c|c|}
\hline & & $\begin{array}{l}\text { Comp. } \\
\text { musical }\end{array}$ & $\begin{array}{l}\text { Comp. } \\
\text { musical/ } \\
\text { sonidos } \\
\text { naturales }\end{array}$ & $\begin{array}{c}\text { Realidad } \\
\text { aumentada }\end{array}$ & $\begin{array}{l}\text { Relato } \\
\text { sonoro }\end{array}$ & $\begin{array}{l}\text { Represen- } \\
\text { tación } \\
\text { sonora }\end{array}$ & $\begin{array}{c}\text { Total } \\
\text { según } \\
\text { el tipo } \\
\text { de } \\
\text { fuente } \\
\text { sonora }\end{array}$ \\
\hline \multirow{12}{*}{$\begin{array}{l}\text { Sonidos } \\
\text { mecánicos }\end{array}$} & Máquinas (genérico) & & & & 1 & & 1 \\
\hline & $\begin{array}{l}\text { Equipamiento } \\
\text { industrial y } \\
\text { de factorías }\end{array}$ & & & & & & \\
\hline & $\begin{array}{l}\text { Medios de } \\
\text { transporte }\end{array}$ & & & & 5 & 4 & 9 \\
\hline & Armas & & & & 4 & 1 & 5 \\
\hline & Trenes, tranvías & & & & 1 & 2 & 3 \\
\hline & $\begin{array}{l}\text { Motores de } \\
\text { combustión interna }\end{array}$ & & & & 5 & 1 & 6 \\
\hline & Aeronaves & & & & 3 & 1 & 4 \\
\hline & $\begin{array}{l}\text { Equipo de } \\
\text { construcción } \\
\text { o demolición }\end{array}$ & & & & & & \\
\hline & $\begin{array}{l}\text { Herramientas o } \\
\text { utensilios mecánicos }\end{array}$ & & & & 14 & 2 & 16 \\
\hline & $\begin{array}{l}\text { Instrumentos de } \\
\text { guerra/destrucción }\end{array}$ & & & & & & \\
\hline & Maquinaria agrícola & & & & & & \\
\hline & Total & 0 & 0 & 0 & 33 & 11 & 44 \\
\hline \multirow{12}{*}{$\begin{array}{l}\text { Sonidos } \\
\text { como indi- } \\
\text { cadores }\end{array}$} & Campanas o gongs & & & & 5 & 2 & 7 \\
\hline & $\begin{array}{l}\text { Instrumentos } \\
\text { de viento }\end{array}$ & 3 & & & 1 & 2 & 6 \\
\hline & Silbidos, silbatos & & & & & & \\
\hline & $\begin{array}{l}\text { Sonidos del tiempo } \\
\text { (relojes) }\end{array}$ & & & & 3 & & 3 \\
\hline & Teléfonos & & & & 4 & & 4 \\
\hline & $\begin{array}{l}\text { Alarmas, sistemas } \\
\text { de alerta }\end{array}$ & & & & 5 & 3 & 8 \\
\hline & $\begin{array}{l}\text { Indicadores de } \\
\text { sucesos futuros }\end{array}$ & & & & & & \\
\hline & $\begin{array}{l}\text { Alertas de redes } \\
\text { sociales }\end{array}$ & & & & & & \\
\hline & $\begin{array}{l}\text { Anuncios de medios } \\
\text { de comunicación }\end{array}$ & & & & & 1 & 1 \\
\hline & $\begin{array}{l}\text { Explosiones o } \\
\text { bombas }\end{array}$ & & & & 2 & & 2 \\
\hline & Advertencias sociales & & & & & & \\
\hline & Total & 3 & 0 & 0 & 20 & 8 & 31 \\
\hline \multirow{4}{*}{ Otros } & Sonidos fuertes & & & & 2 & & 2 \\
\hline & Silencio & & & & & & \\
\hline & $\begin{array}{l}\text { Efectos } \\
\text { desconocidos }\end{array}$ & & & & & & \\
\hline & Total & & & & 2 & & 2 \\
\hline \multicolumn{2}{|c|}{ Total fuentes de sonido } & 14 & 4 & 2 & 159 & 102 & 281 \\
\hline
\end{tabular}

Fuente: Elaboración propia. 
A continuación, se realizará un conteo del total de fuentes sonoras empleadas en los paisajes. Con él, se podrá visualizar la distribución de los sonidos en función de la clasificación del sonido de Schafer (1993) como el uso de cada agrupamiento en función de la categoría sonora en la que es empleada, así como el conteo total de los sonidos para ambas funciones.

Tabla 3. Tabla de frecuencia de los sonidos empleados en los paisajes sonoros en función de su categoría y su correspondiente distribución.

\begin{tabular}{lcccccc}
\hline & $\begin{array}{c}\text { Composición } \\
\text { musical }\end{array}$ & $\begin{array}{c}\text { Comp. } \\
\text { musical/ } \\
\text { sonidos } \\
\text { naturales }\end{array}$ & $\begin{array}{c}\text { Realidad } \\
\text { aumentada }\end{array}$ & $\begin{array}{c}\text { Relato } \\
\text { sonoro }\end{array}$ & $\begin{array}{c}\text { Repre- } \\
\text { sentación } \\
\text { sonora }\end{array}$ & Total \\
\hline $\begin{array}{l}\text { Sonidos naturales } \\
\text { Sonidos humanos }\end{array}$ & 2 & 2 & 0 & 23 & 42 & 69 \\
$\begin{array}{l}\text { Sonidos y } \\
\text { sociedad }\end{array}$ & 1 & 0 & 1 & 36 & 17 & 55 \\
$\begin{array}{l}\text { Sonidos } \\
\text { mecánicos }\end{array}$ & 0 & 2 & 1 & 45 & 24 & 80 \\
$\begin{array}{l}\text { Sonidos como } \\
\text { indicadores }\end{array}$ & 3 & 0 & 0 & 33 & 11 & 44 \\
$\begin{array}{l}\text { Otros } \\
\text { Total }\end{array}$ & 0 & 0 & 0 & 20 & 8 & 31 \\
& 14 & 4 & 2 & 159 & 102 & 281 \\
\hline
\end{tabular}

Fuente: elaboración propia.

Como se puede comprobar en la Tabla 3, abundan mayormente los sonidos asociados a sonidos y sociedad -derivados de la humanización de entornos-, seguidos por los sonidos naturales y los sonidos realizados por los humanos. Asimismo, también se puede ver como los relatos sonoros son aquellos que agrupan mayor cantidad de clips de sonido, si bien es cierto que son el $45.5 \%$ de los paisajes sonoros totales. Con ello, podremos caracterizar qué tipo de sonidos son de los que se sirven para obtener el fin que desean a través de sus paisajes sonoros.

\section{Conclusiones}

Los avances tecnológicos de las últimas dos décadas y la generalización de internet han posibilitado que herramientas como Audacity lleguen a un gran público puesto que, gracias a su facilidad de uso, posee multiplicidad de funciones y aporta numerosas ventajas extrapolables a una gran diversidad de entornos y áreas de estudio, entre ellas, la creación de material educativo.

En este caso, el paisaje sonoro -comprendido desde una visión holística (Schafer, 1993)- es el campo donde los entornos sonoros se evalúan, analizan y clasifican como una composición de eventos sonoros procedentes del medio que rodea al ser humano. La creación de paisajes sonoros es una forma de representación de cómo un individuo comprende la realidad y cómo es capaz de expresarla a través de sus destrezas y herramientas. Como explica Ariza-Pomareta, la "elección de estos sonidos ofrece al oyente una percepción acústica que permite establecer relaciones 
nítidas con la imagen mental a la que puede corresponder" (2015, p. 74). Por tanto, y dejando de lado análisis físicos, contemplamos el diseño de paisajes sonoros como una forma de comprensión del entorno, en cómo la teoría del paisaje sonoro atiende a la semiótica ya que los procesos socioculturales de una época o generación son transversales en los individuos de esta, siendo coincidentes en gustos y modas (Cárdenas-Soler y Martínez-Chaparro, 2015).

La división entre unas y otras categorías son trasversales culturalmente y parece que existen relaciones estables entre semiótica y las categorías sonoras. En términos generales, las personas tienen preferencias por los sonidos naturales frente a los urbanos (Brown, Kang y Gjestland, 2011; Yang y Kang, 2005) y hacia los sonidos humanos frente a los de origen mecánico (Axelsson, Nilsson y Berglund, 2010; Dubois, Guastavino, Riambault, 2006). Estos resultados son coincidentes con los obtenidos con nuestra muestra, ya que en ella los sonidos de origen natural tienen la mayor representación -se excluye la categoría de sonidos y sociedad, ya que poseen fuentes mixtas, tanto naturales como humanas (Schafer, 1993)-, seguidas por las fuentes humanas y, por último, las de origen mecánico.

Además, estas atribuciones subjetivas también están relacionadas con la propia percepción del entorno. A través de las diferentes propuestas obtenidas hemos podido comprobar cómo los participantes tienden a separar las fuentes de origen humano y humanizadas de aquellas que son de origen natural y tienden a situar las primeras en los relatos sonoros mientras que las segundas en las representaciones sonoras. Estas agrupaciones no parecen ser inocuas, sino que aparentan correlacionar con resultados similares en los que se relacionan paisajes sonoros en función del estado emocional evocado. Sudarsono, Nitidara y Sarwono (2018) comprueban que la atribución perceptiva de la situación dinámica de los sonidos se enfrenta a la percepción de estabilidad o relajación de las fuentes de sonido. Aquellos sonidos etiquetados como dinámicos -sonidos bulliciosos de origen humano, el tráfico humano o eventos sonoros- son opuestos a los etiquetados como relajados o estables -música- y así los categorizan los participantes, situando los primeros en los relatos sonoros -donde encontramos los relatos, en los que se suceden acciones de forma continuada- frente a las representaciones sonoras; más próximas a la evocación de un lugar o situación-en su mayoría, de connotaciones naturales en entornos sin humanizar-.

Por tanto, podríamos emplear el término de paisaje sonoro como un lugar para crear representaciones de "el entorno acústico tal como lo perciben los humanos" (Brown, Kang y Gjestland, 2011). Esta aproximación a la creación de paisajes sonoros es una fórmula en la que el participante sintetiza sus conocimientos, elaborando un discurso en el que se pueden observar sus tendencias hacia connotaciones perceptivas o imaginarias reflejadas en un paisaje sonoro. Además, la actividad de creación de paisajes sonoros cada vez está cobrando más importancia con usos y fines de lo más variopintos. Sin embargo, la creación de paisajes sonoros puede ser empleada en muchos otros ámbitos (Randall, 2012; Reich, 2018; Wakefield y France, 2015), porque representan las aproximaciones del alumno a su contexto más próximo y su simbolización a través de las herramientas disponibles que éste posee.

Por último, a tenor de la revisión de la literatura existente se constató que Audacity es una herramienta idónea para la creación de material educativo desde numerosas aproximaciones, ya no únicamente desde la vertiente de la creación de paisajes sonoros para potenciar, entre otras, las habilidades creativas e imaginativas de tanto el alumnado como del profesorado, sino también como herramienta para 
mejorar el propio autoconcepto y autoestima del alumnado (Sichivitsa, 2017), las capacidades auditivas (Ibáñez Barrachina y De Vicente-Yagüe Jara, 2014), sus habilidades comunicativas (Galán Camano, 2018; Sousa y Bessa, 2008) contribuir al bienestar y estado de salud en general (Karageorghis, 2017) e incluso para adentrarse en el campo de la realización de proyectos de investigación (Miguel Orellana,2014; Sankupellay, Tosey, Truskinger y Roe, 2015; Sáenz, 2018). Por todo ello y vista la polivalencia que ofrece esta herramienta, como trabajo de futuro se antojaría sumamente interesante abordar su estudio en profundidad, ya no únicamente desde una vertiente genérica como herramienta de creación de materiales educativos, sino entrando ya en la particularidad de cada uno de sus usos posibles.

\section{Referencias}

Amundsen, C.P., y Belmonte, C. (2018). A how to podcast in Archaeology: A guide for archaeologists doing public outreach. Advances in Archaeological Practice, 6(Special Issue 3), 259-266. https://doi.org/ 10.1017/aap.2018.20

Ariza-Pomareta, J. (2015). El paisaje sonoro como cápsula del tiempo: Un proyecto creativo basado en los sonidos de la primera estación de trenes de Cuenca. Arte, Individuo y Sociedad, 27(1), 65-79. https://doi.org/10.5209/rev_ARIS.2015.v27 .n1.43741

Axelsson, Ö., Nilsson, M.E., y Berglund, B. (2010). A principal components model of soundscape perception. The Journal of the Acoustical Society of America, 128(5), 2836-2846.

https://doi.org/10.1121/1.3493436

Bairros Soares, A., Vielmo Miranda, P., y Barin Smaniotto, C. (2018). Potencial pedagógico del podcast en educación superior. REDIN, 7(1), 1-10. Recuperado de http://seer.faccat.br/index.php/redin/articl e/view/1078

Bell, S., y Buri, D. (2017). That sounds easy! Simple audio recording and editing for instructional podcasts. En: ARCLIB 2017 Conference. University of Bath, Inglaterra. Recuperado de https://strathprints.strath.ac.uk/id/eprint/6 3856

Beltrán, P., y Rodríguez, C. (2017). Vitalinux y software libre para el profesorado de Matemáticas. Entorno abierto, 16, 7-12.

Brown A.L., Kang, J., y Gjestland, T. (2011). Towards standardization in soundscape preference assessment. Applied Acoustics, 72

387-392. https://doi.org/10.1016/j.apacoust.2011.01 .001

Cárdenas-Soler, R.N. y Martínez-Chaparro, D. (2015). El paisaje sonoro, una aproximación teórica desde la semiótica. RIDI, 5(2), 129-140 https://doi.org/10.19053/20278306.3717

Cepeda, O., Gallardo, J.M., y Rodríguez, J. (2017). La evaluación de los materiales didácticos digitales. Revista Latinoamericana de Tecnología Educativa RELATEC, 16(2), 79-95. https://doi.org/10.17398/1695288X.16.2.79

Chao-Fernández, R., Felpeto Guerrero, A., y Vázquez-Sánchez, R. (2018). MOOC Música para el siglo XXI: dificultades y evaluación de la experiencia. En J. Valverde-Berrocoso (Ed.), Campus digitales en la Educación Superior. Experiencias e investigaciones, 509-519

Dias, M.A., Carvalho P.S., y Rodrigues, D. (2016). How to study the Doppler effect with Audacity software. Physics Education, 51(3), 1-5. https://doi.org/10.1088/00319120/51/3/035002

Dubois, D., Guastavino, C., y Riambault, M. (2006). A cognitive approach to urban soundscapes: Using verbal data to access everyday life auditory categories. Acta Acustica United with Acustica, 92(6), 865874.

Ferretti, U. (2006). Sonido ambiental, entorno sonoro y música. En XVI Congresso da Associação Nacional de Pesquisa e Pósgraduaçãoem Música (ANPPOM), 781-784. Universidade de Brasilia, Brasilia, Brasil.

Forbes, D., y Khoo, E. (2015). Voice over Distance: A Case of Podcasting for 
Learning in Online Teacher Education. Distance Education, 36(3), 335-350. http:// dx.doi.org/10.1080/01587919.2015.10840 74

Galán Camacho, F. (2018). Aplicaciones del podcast en el aula de ELE. E-eleando, ELE en Red, 8. Alcalá, Madrid: Universidad de Alcalá, Servicio de Publicaciones. http://hdl.handle.net/10017/34682

Gailey, A. (2015). Signal Frequency Spectra with Audacity ${ }^{\oplus}$. The Physics Teacher, 53(239), https://doi.org/10.1119/1.4914568

Godofredo, J.L., León, T., y Liern, V. (2010). Música e informática en las clases de matemáticas. Suma, 64, 113-118.

Hurtado, A., y Botella, A.M. (2017). Realidad aumentada y Realidad Virtual aplicadas a la didáctica del paisaje: resultados del proyecto L'Hort 2.0, en el Simposio: Las Tics como herramienta docente en el aula de educación superior. XIV Foro Internacional sobre la evaluación de la calidad de la investigación y de la educación superior (FECIES). Granada

Ibáñez Barrachina, J., y de Vicente-Yagüe Jara, M.I. (2014). Didáctica de la afinación en los instrumentos de metal: ciencia, música y tecnología en las Enseñanzas Artísticas. Revista Internacional de Tecnologías en la Educación, 1(2), 2-11. https://dialnet.unirioja.es/servlet/articulo? codigo $=6367385$

Karageorghis, C.I. (2017). Applying music in exercise and sport. Londres, Reino Unido: Human Kinetics.

Krippendorff, K. (1997). Metodología de análisis de contenido. Teoría y práctica. Barcelona: Paidós Comunicación.

Lee, J., Hicks, D., Henriksen, D., Mishra, P., Cain, W., Fahnoe, C., y Terry, C. (2015). Historical Soundscapes for Creative Synthesis. TechTrends, 59(5), 4-8. https://doi.org/10.1007/s11528-015-08826

Lopes, P., Liapis, A., y Yannakakis, G.N. (2017). Modelling affect for horror soundscapes. IEEE Transactions on Affective Computing, 10(2), 209-222. https://doi.org/10.1109/TAFFC.2017.26954 60
Lund, B., Brandt, H., y Swensen, H. (2018) (2018). Students developing representational competence as producers with and of augmented reality in science. NORDINA, 14(2), 138-153. https://doi.org/10.5617/nordina.6163

Marson, I., y Santos, A. (2010). Internet e práctica pedagógica. Possibilidades para a doçencia da língua inglesa. Revista ecurriculum, 5(2), 1-26.

Miguel Orellana, A. (2014). Estudio de la bioacústica del zorzal andino (Turdus chiguanco) con la aplicación del software Audacity, v. 2.0. Apuntes de Ciencia \& Sociedad, 4(2), 259-266. http://dx.doi.org/ $10.18259 /$ acs. 2014030

Moscoso, P., Peck, M., y Eldridge, A. (2018). Emotional associations with soundscape reflect human-environment relationships. Journal of Ecoacoustics, 1, 1-19. https://doi.org/10.22261/JEA.YLFJ6Q

Nielsen, B.L., Brandt, H., y Swensen, H. (2018). Augmented Reality in science education affordances for student learning. NorDiNa, 14(2), 138-153. http://dx.doi.org/10.5617/nordina.2399

Palmese, C., Carles, J.L., y Alcázar, A. (2010). Un acercamiento pedagógico al paisaje sonoro. Paisajes sonoros de Cuenca. España: Servicio de publicaciones de la Universidad de Castilla-La Mancha.

Ponce de León Barranco, L., y Lago Núñez, P. (2017). Sacar partido a las TIC. Preparación y reproducción de fragmentos sonoros con Audacity y Sonic Visualiser. Eufonía: Didáctica de la música, (72), 47-52. https:// dialnet.unirioja.es/servlet/articulo? codigo $=6070863$

Purves, R. (2018). Technology and the Educator. En G.E. McPherson y G.F. Welch (Eds.), Creativities, Technologies, and Media in Music Learning and Teaching: An Oxford Handbook of Music Education, 5, 143-161 Oxford, USA: Oxford University Press.

Randall, A. (2012). 10 creative uses for Audacity that you probably haven't thought of. Make Use Of. Recuperado de https://www.makeuseof.com/tag/10creative-audacity-havent-thought/

Reich, M. A. (2018). Soundscape Composition: Music as Environmental Activism. (Trabajo de fin de grado). Faculta de Música, 
Universidad de Puget Sound, Estados Unidos. Recuperado de https://soundideas.pugetsound.edu/hono rs_program_theses/27

Sáenz Abarzuza, I. (2018). La investigación del timing en la interpretación. El software como herramienta en el análisis de la música clásica tonal. El Oído Pensante, 6(1), 73-90. https://dialnet.unirioja.es/servlet/articulo? codigo $=6316483$

Sales Romeu, C., Roig Barrio, A. y Marín Suelves, D. (2019). Análisis DAFO de herramientas tecnológicas para el área de Música. DIM: Didáctica, Innovación y Multimedia 37. Recuperado de: https://www.raco.cat/index.php/DIM/articl e/view/356988

Sánchez Satoca, C. (2012). Grabación y edición digital de audio para fomentar la expresión oral y la comprensión auditiva en el aula. En P.J. Molina Muñoz (Coord.), Actas de las IV Jornadas de Formación para Profesores de Español en Chipre, 5156. Nicosia, Chipre; Universidad de Chipre.

Sankupellay, M., Towsey, M., Truskinger, A., y Roe, P. (2015). Visual fingerprints of the acoustic environment: The use of acoustic indices to characterise natural habitats. 2015 Big Data Visual Analytics (BDVA), Hobart, TAS. 2015, 1-7. https://doi.org/10.1109/BDVA.2015.73143 06

Saulo Bolaños, C. (2004). Audio digital con Audacity 1.2.0. Todo linux: la revista mensual para entusiastas de GNU/LINUX, (43), 40-43. https://dialnet.unirioja.es/servlet/articulo? codigo $=3237920$

Schafer, R.M. (1993). The soundscape: our sonic environment and the tuning of the world. Rochester, USA: Destiny Books.
Sichivitsa, V. (2007). Audacity in Vocal Improvisation: Motivating Elementary School Students through Technology. Teaching Music, 14(4), 48. https://eric.ed.gov/?id=EJ754960

Sikora, M., Grčić, Đ., y Russo, M. (2016). A tool for soundscape auralization of ancient archaeological sites. 7 th $A A A A$ Congress on Sound and Vibration. Recuperado de https://bib.irb.hr/datoteka/838151.AAAA_ 16.pdf

Silva, M.A.A. (2011). Imagens sonoras do ambiente: educação ambiental e ensino de música. Relato de uma pesquisa participante no ensino superior de licenciatura em música. European Review of Artistic Studies, 2(2), 1-12.

Smith, K. H. (2011). Using Audacity and One Classroom Computer to Experiment with Timbre. General Music Today, 24(3), 23-27. Recuperado de https://doi.org/10.1177/104837131038542 1.

Sudarsono A.S, Nitidara, N.P.A., y Sarwono, J. (2018). The relationship between sound source and urban soundscape. En IOP Conf. Series: Journal of Physics: Conf. Series 1075, 1-5. Bali, Indonesia: Association of Acoustics and Vibration \& Society of Acoustics Singapore. https://iopscience.iop.org/article/10.1088/ 1742-6596/1075/1/012033

Wakefield, K., y France, D. (2015). Bringing digital stories into assessment. Planet, 23(1), 63-67. https://doi.org/10.11120/plan.2010.00230 063

Yang, W., y Kang J. (2005). Soundscape and Sound Preferences in Urban Squares: A Case Study in Sheffield. Journal of Urban Design, 10(1), 61-80 https://doi.org/10.1080/135748005000623 95 
